\author{
H. Farhangkhoee $\cdot$ Z. A. Khan $\cdot$ Y. Barbin $\cdot$ \\ S. Chakrabarti
}

\title{
Glucose-induced up-regulation of CD36 mediates oxidative stress and microvascular endothelial cell dysfunction
}

Received: 21 October 2004 / Accepted: 26 February 2005 / Published online: 25 May 2005

(C) Springer-Verlag 2005

\begin{abstract}
Aims/hypothesis: Hyperglycaemia-induced oxidative stress is implicated in the pathogenesis of chronic diabetic complications. Glucose-mediated oxidation of LDL may result in increased oxidative stress and vascular endothelial cell dysfunction via interaction with a cell surface scavenger receptor, CD36. In this study, we investigated the role of $\mathrm{CD} 36$ in cultured microvascular endothelial cells (MVECs) and in the heart by using an animal model of chronic diabetes. Methods: Cultured MVECs were subjected to varying glucose concentrations and assayed for alteration in CD36 gene expression and protein levels. To assess for oxidised LDL (ox-LDL) uptake, MVECs exposed to low and high glucose were treated with ox-LDL $(80 \mu \mathrm{g} / \mathrm{ml})$, a ligand for CD36. Haem oxygenase-1 (HO-1) and endothelin-1 (ET-1) induction, as well as oxidative stress were determined. The role of glucose-induced CD36 alteration in ox-LDL uptake was also assayed following post-transcriptional CD36 gene silencing. For in vivo studies, CD36 mRNA and oxidative DNA and protein damage were measured in heart tissues of 1-month-old diabetic Sprague-Dawley rats. Results: We found that glucose increased CD36 mRNA and protein levels in MVECs. High levels of glucose also augmented ox-LDL uptake, in association with increasing HO-1 and ET-1 mRNA levels. CD36 gene silencing prevented glucose-induced CD36 alteration, reduced ox-LDL uptake,
\end{abstract}

H. Farhangkhoee $\cdot$ Z. A. Khan · Y. Barbin $\cdot$ S. Chakrabarti Department of Pathology, University of Western Ontario, London, ON, N6A 5C1, Canada

\section{Z. A. Khan}

Department of Surgery, Children's Hospital Boston and Harvard Medical School,

Boston, MA, USA

S. Chakrabarti $(\bowtie)$

Department of Microbiology \& Immunology,

University of Western Ontario,

London, ON, Canada

e-mail: schakrab@uwo.ca

Tel.: +1-519-6858500

Fax: +1-519-6613370 and prevented HO-1 and ET-1 up-regulation. Similar to in vitro studies, diabetic heart tissues exhibited increased CD36 mRNA levels and increased oxidative DNA and protein damage. Conclusions/interpretation: Our results provide evidence that up-regulation of CD36 may have a role in increasing oxidative stress in MVECs and the heart in chronic diabetes.

Keywords Cardiomyopathy - CD36 - Diabetic complications · Endothelin-1 Haem oxygenase · Oxidative stress $\cdot$ Oxidised LDL $\cdot$ Scavenger receptor

Abbreviations 8-OHdG: 8-hydroxy-2'-deoxyguanosine . EC: endothelial cell - ET-1: endothelin-1 - HO-1: haem oxygenase-1 - HG: high glucose $\cdot$ LG: low glucose MVEC: microvascular endothelial cell - ox-LDL: oxidised LDL $\cdot$ siRNA: small interfering RNA - SR: scavenger receptor $\cdot$ STZ: streptozotocin

\section{Introduction}

In spite of the improvements in therapeutic modalities, diabetes and its complications account for significant morbidity and mortality $[1,2]$. Long-standing diabetes leads to structural and functional alterations in both the micro- and macrovasculature [3, 4]. Diabetic cardiomyopathy has been recognised as a degenerative microvascular complication of chronic diabetes $[5,6]$. Understanding the pathogenesis of diabetic cardiomyopathy is still obscure; however, oxidative stress has been suggested to be involved in the development and progression of diabetes-induced cardiomyopathy [7].

Hyperglycaemia caused by chronic diabetes is an important mediator in augmenting reactive oxygen species [8]. Oxidative stress has been shown to occur in diabetic patients before the presentation of overt chronic complications, including cardiomyopathy [7]. The generation of oxidative stress in diabetes could be due to increased generation of free radicals and nitric oxide $[9,10]$. Free radicals are produced in all organisms as a result of increased 
oxidative stress or altered redox balance. Unpaired electrons from these free radicals can modify and thus alter the function of proteins, DNA, fatty acids and lipids [11]. The LDLs are very sensitive to these free radical-mediated peroxidation reactions, yielding oxidised LDL (ox-LDL) molecules [12].

Modified proteins such as ox-LDLs and AGE products including glycated LDLs accumulate over time in diabetic patients [13-15]. Alterations of these proteins may interfere with their recognition by native receptors such as LDL receptor. Nonetheless, these modified proteins can interact with greater affinity with a group of multifunctional transmembrane proteins named scavenger receptors (SRs) [16, 17]. Recent studies have demonstrated an important role of SRs in a number of human diseases [18]. A prominent member of the SR family is CD36 [19]. CD36 was first identified as a surface glycoprotein on platelets $[19,20]$. Since then, it has been shown to be expressed on macrophages, adipocytes and other cell types including microvascular endothelial cells (MVECs) [21]. High-affinity binding of ox-LDLs and AGE-modified proteins to CD36 suggests that alterations of CD36 could potentially be involved in diabetic complications, including cardiomyopathy $[22,23]$.

In the present study, we used cultured MVECs, the primary target of sustained hyperglycaemia, to elucidate whether up-regulation of CD36 underlies increased ox-LDL uptake and subsequent endothelial dysfunction. Furthermore, we investigated whether diabetes leads to an alteration of CD36 in the heart and whether such an alteration is associated with diabetes-induced oxidative stress.

\section{Materials and methods}

In vitro studies Human MVECs (Clonetics, Walkersville, MD, USA) were cultured with endothelial growth medium (Clonetics) as previously described [24]. To determine the optimal high glucose (HG) concentration, sub-confluent cells were incubated with $10,15,25$ or $35 \mathrm{mmol} / \mathrm{l}$ D-glucose. Following a 24-h treatment period, total RNA was extracted and subjected to CD36 mRNA expression by realtime RT-PCR. Subsequent experiments were conducted with treatment of cells exposed to low glucose (LG) or empirically determined HG levels. These cells were subjected to CD36 mRNA and protein expression by real-time RT-PCR and western blotting, respectively.

In order to determine whether alteration of CD36 coincides with functional significance, cells exposed to LG and HG were treated with ox-LDL. Ox-LDL was prepared as described before [25]. Briefly, human LDL (Sigma-Aldrich Canada, Oakville, ON, Canada) was resuspended in PBS and incubated in the presence of $5 \mu \mathrm{mol} / 1 \mathrm{CuSO}_{4}$ in order to oxidise the LDL molecules. Following an incubation period of $3 \mathrm{~h}$, the ox-LDL samples were extensively dialysed overnight with repeated buffer changes to remove $\mathrm{CuSO}_{4}$. Uptake of ox-LDL was assessed by immunocytochemistry following exposure of endothelial cells (ECs) to $80 \mu \mathrm{g} / \mathrm{ml}$ ox-LDL. This concentration of ox-LDL has been well established in EC culture studies [26]. Cells exposed to ox-LDL were also subjected to mRNA analyses for the oxidative stress marker, haem oxygenase-1 (HO-1) [27, 28] and vasoactive peptide, endothelin-1 (ET-1). ET alteration has been well established in chronic diabetic complications and vascular endothelial dysfunction [29].

CD36 gene silencing To establish the role of CD36 in mediating ox-LDL uptake, MVECs were transfected with small interfering RNA (siRNA) targeted to CD36 mRNA (Ambion, Austin, TX, USA). Cultured MVECs were transfected with $100 \mathrm{nmol} / 1$ CD36 siRNA using siPORT Lipid transfection media (Ambion). Following a 24-h incubation period, cells were cultured for another $24 \mathrm{~h}$ in either 5 or $25 \mathrm{mmol} / 1$ glucose, as well as treated with the CD36 ligand, ox-LDL. All siRNA experiments included transfection of ECs with siRNAs which have no sequence homology (negative control transfection) with human genome (Ambion). To measure CD36 siRNA transfection efficiency, CD36 mRNA levels were assayed for using real-time RT-PCR. Ox-LDL uptake was determined by immunohistochemical analysis using ox-LDL antibody (1:500).

In vivo studies Male Sprague-Dawley rats (Charles River Canada, St Constant, QC, Canada) weighing approximately $250 \mathrm{~g}$ were made diabetic by a single i.v. injection of streptozotocin (STZ; $65 \mathrm{mg} / \mathrm{kg}$; Sigma-Aldrich) [24]. Ageand sex-matched controls were given an equal volume of citrate buffer. Diabetes was confirmed by measuring blood glucose levels (Surestep; Lifescan, Burnaby, BC, Canada) $48 \mathrm{~h}$ after the injection of STZ. Animals were also monitored for glucosuria and ketonuria (Uriscan Gluketo; Yeong Dong Co., Seoul, Korea) and given small daily doses (0.1$3.0 \mathrm{U}$ ) of ultralente insulin (Novo Nordisk, Princeton, NJ, USA) to prevent ketoacidosis [24]. Following 1 month of treatment, rats were killed and heart tissues were removed. Prior to killing, blood was collected from the rats to assay for $\mathrm{HbA}_{1} \mathrm{c}$ levels (Glycotest; Pierce, Rockford, IL, USA). Heart tissues were sectioned and either snap-frozen in liquid nitrogen for gene expression analyses or embedded in paraffin for immunohistochemical analysis. The University of Western Ontario Council on Animal Care Committee formally approved all experimental protocols.

Real-time RT-PCR Total RNA from cultured ECs and heart tissues were isolated as previously described [24]. Briefly, RNA was extracted using TRIzol (Invitrogen, Burlington, ON, Canada) with isopropanol precipitation. Following extraction, RNA samples were subjected to DNAse treatment to degrade any contaminating DNA in the samples. Purity was assessed by measuring OD 260:280 nm.

cDNA was synthesised using $3 \mu \mathrm{g}$ total RNA with oligo(dT) primers and Superscript-II MMLV-reverse transcriptase (Invitrogen). Real-time RT-PCR was performed in the LightCycler (Roche Diagnostics Canada, Laval, QC, Canada) as previously described [24]. The reaction mixture (20 $\mu \mathrm{l}$ total volume) consisted of $10 \mu \mathrm{l} \mathrm{SYBR}$ Green Taq ReadyMix (Sigma-Aldrich), $1.6 \mu 125 \mathrm{mmol} / 1 \mathrm{MgCl}_{2}, 1 \mu \mathrm{l}$ of each forward and reverse $10 \mu \mathrm{mol} / 1$ primers, $4.4 \mu \mathrm{l}$ 
$\mathrm{H}_{2} \mathrm{O}$, and $2 \mu \mathrm{l} \mathrm{cDNA}$ template. The primer sequences and PCR temperature profiles for $\beta$-actin (human/rat) and HO1 (rat) were assayed as previously described [24, 30, 31]. The primer sequences for CD36 are 5'TAATGGCACA GATGCAGCCT3' and 5'ACAGCATAGATGGACCTGC AA3' (human) and 5'GAGAACTGTTATGGGGCTAT3' and 5'TTCAACTGGAGAGGCAAAGG3' (rat). The PCR temperature profiles for $\mathrm{CD} 36$ are similar to the $\beta$-actin PCR profiles [30]. The PCR reaction mixture for ET-1 consisted of $2.5 \mu \mathrm{l} 10 \times \mathrm{PCR}$ Buffer (Invitrogen), $1.25 \mu \mathrm{l} 5$ $\mathrm{mmol} / \mathrm{l} \mathrm{dNTP}, 1.2 \mu \mathrm{l} 50 \mathrm{mmol} / 1 \mathrm{MgCl}_{2}, 1 \mu \mathrm{l}$ primers, $9.8 \mu \mathrm{l}$ $\mathrm{H}_{2} \mathrm{O}, 2 \mu \mathrm{l} \mathrm{cDNA}$, and $0.75 \mu \mathrm{l} 15 \mathrm{mmol} / \mathrm{l}$ Taqman probe Primer sequences and PCR temperature profiles for ET-1 were assayed as previously described [24]. The data were
Fig. 1 Real-time RT-PCR amplification of human CD36 showing a PCR amplification curves b melting curve analysis (MCA) of post-PCR products, and $\mathbf{c}$ mRNA expression following 24-h incubation with varying glucose concentrations. Each trace represents a human MVEC cDNA sample; all PCR reactions were coupled to MCA to determine specificity of amplification. CD36 is expressed as ratio of target to $\beta$-actin (relative to $10 \mathrm{mmol} / 1$ glucose); $n=4 /$ treatment; ${ }^{*} p<0.05 \mathrm{com}$ pared with $10 \mathrm{mmol} / \mathrm{l}$ glucose a

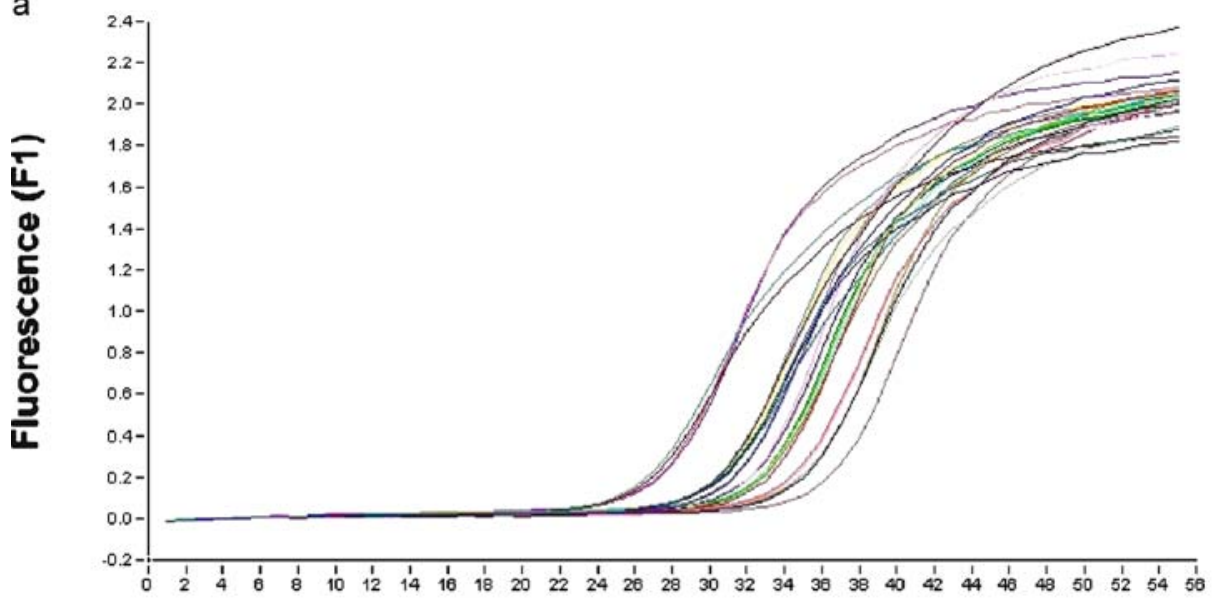

Cycle number

b

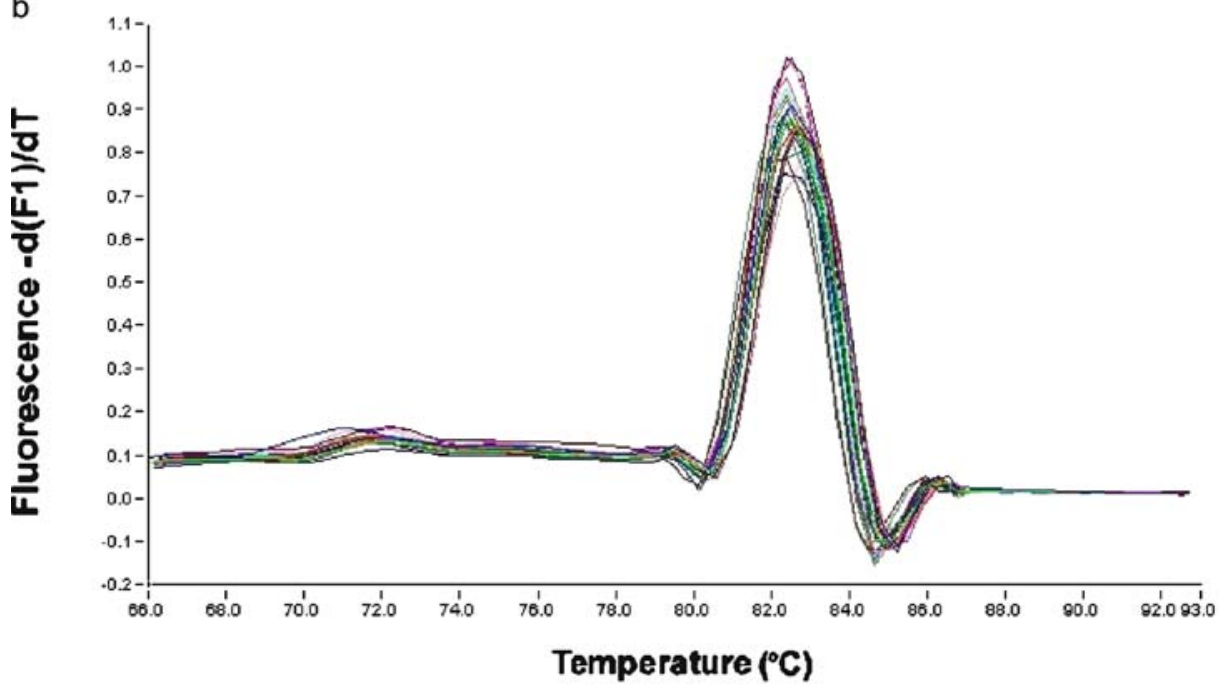

C

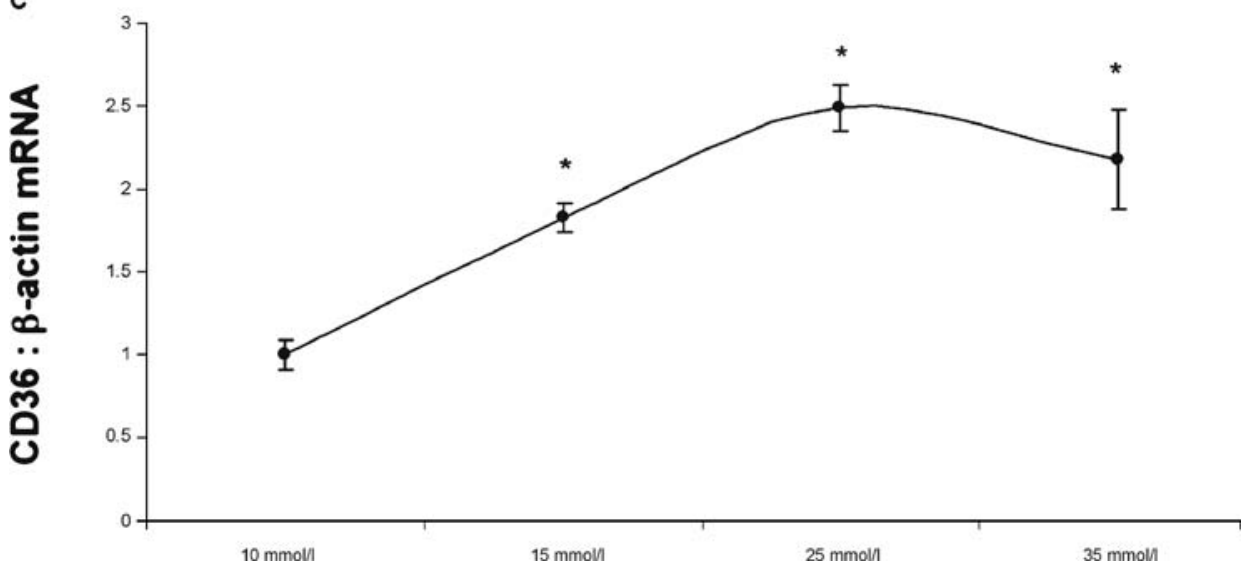


normalised using the housekeeping gene, $\beta$-actin, to account for differences in reverse transcription efficiencies and in the amount of template in the reaction mixtures.

Western blotting Total proteins from ECs were isolated and quantified according to well-established methodologies [24]. Briefly, cells were homogenised in complete lysis buffer $(\mathrm{NaCl} 0.877 \mathrm{~g}$, deoxycholate $1 \mathrm{~g}, 1 \mathrm{mmol} / 1 \mathrm{Tris}-$ $\mathrm{HCl}$ [pH 7.5] $5 \mathrm{ml}$, Triton X-100 $1 \mathrm{ml}$, and $10 \%$ sodium dodecyl sulphate $1 \mathrm{ml}$; volume adjusted to $100 \mathrm{ml}$ using $\mathrm{ddH}_{2} \mathrm{O}$ ) and protease inhibitor. Total proteins were quantified by a BCA protein assay kit (Pierce). Western blotting was performed by polyclonal anti-human CD36 antibody (1:1000; Cayman Chemicals, Ann Arbor, MI, USA) followed by secondary antibody conjugated with horseradish peroxide. An ECL-Plus Western Blotting Detection kit (Amersham Pharmacia Biotechology, Piscataway, NJ, USA) was used for detection.

Immunochemical analyses Paraffin-embedded heart tissues were stained for 8-hydroxy-2'-deoxyguanosine (8-OHdG) and nitrotyrosine as described previously [30]. Briefly, 5 $\mu \mathrm{m}$ sections were transferred to positively charged slides. Monoclonal anti-mouse 8-OHdG (1:150; Chemicon Lab., Temecula, CA, USA ) and monoclonal anti-mouse nitrotyrosine (1:75; Cayman Chemicals) were used for staining. Secondary antibodies conjugated with horseradish peroxidase (Bio-Rad Laboratories, Hercules, CA, USA) were used to produce signals from the chemiluminescent substrate, diaminobenzidine (Amersham Pharmacia Biotechnology). Negative controls included incubation with PBS without primary antibody. Specificity of the antibodies was confirmed by blocking tissue sections with $10 \%$ horse serum. The experiments were performed in triplicate and slides were read by two investigators unaware of the particular treatment. 8-OHdG immunoreactivity was expressed as the number of positive cardiomyocytes in ten random

fields containing approximately 100 cells. Nitrotyrosine was evaluated by relative cytoplasmic staining intensity. The data are expressed as percentage of total cell.

For immunocytochemistry, cultured vascular ECs were briefly trypsinised and seeded in 12-well plates containing coverslips. Cells were cultured in growth media for $24 \mathrm{~h}$ to allow attachment. Following cell attachment, all treatments were carried out in serum-free media as described above. Anhydrous ethanol was used to fix the cells. Ox-LDL (1: 500; Biodesign International, Saco, ME, USA) and 8-OHdG (1:400; Chemicon) immunochemical analyses were carried out essentially the same as described for heart tissues.

Statistical analysis The data are expressed as means \pm SEM and were analysed by ANOVA followed by Student's $t$-test. Differences were considered statistically significant at values of $p<0.05$.

\section{Results}

Glucose induces up-regulation of CD36 in MVECs We used vascular ECs to elucidate whether glucose-induced alteration of CD36 may mediate increased oxidative stress. Cultured MVECs were exposed to varying glucose concentrations and assayed for CD36 mRNA alteration. Our data show up-regulation of CD36 mRNA by glucose in a dose-dependent manner (Fig. 1). The greatest change in transcript levels was observed with $25 \mathrm{mmol} / \mathrm{l}$ glucose; thus, subsequent experiments were carried out by treating cells with either 5 or $25 \mathrm{mmol} / \mathrm{l}$ glucose. In order to determine whether mRNA levels coincide with protein expression, we used the western blotting technique. Here we show that, in parallel to mRNA, CD36 protein expression is augmented by exposure of ECs to $25 \mathrm{mmol} / \mathrm{l}$ glucose (Fig. $2 \mathrm{a}, \mathrm{b} ; p<0.05$ ).

Fig. 2 Glucose-induced upregulation of $\mathrm{CD} 36$ protein expression in MVECs showing a representative immunoblot of CD36 and $\beta$-actin, and b semiquantitative analysis of CD36 protein expression (relative to $\beta$-actin). ${ }^{*} p<0.05 \mathrm{HG}$ compared with LG; $n=4 /$ treatment a

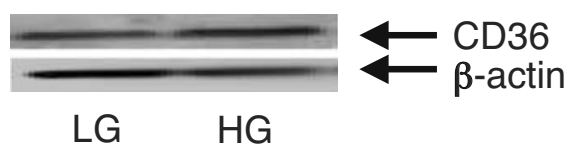

b

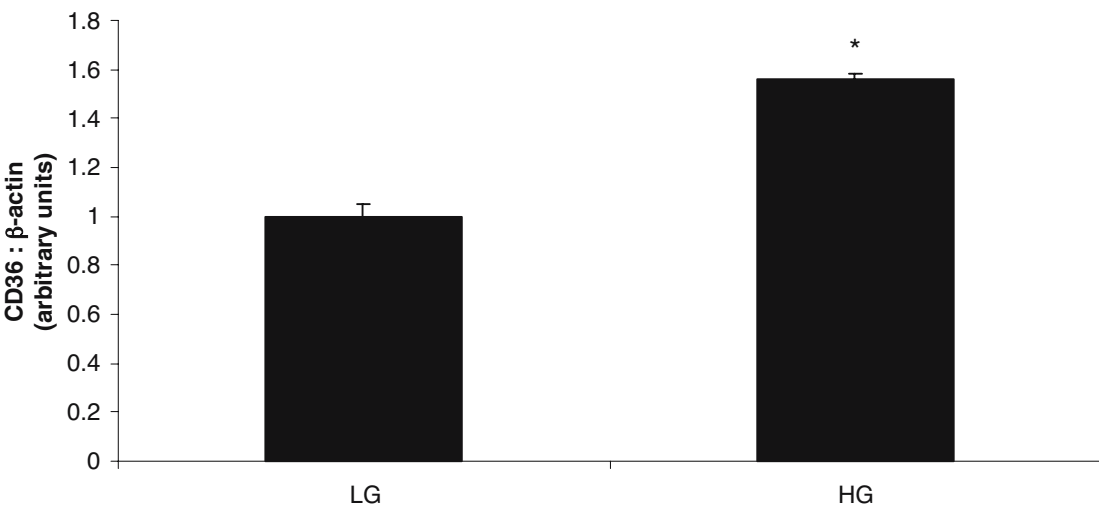


Fig. 3 Ox-LDL uptake in MVECs exposed to $\mathbf{a} \mathrm{LG}$ and $\mathbf{b}$ HG. Cells in LG or HG glucose were treated with $80 \mu \mathrm{g} / \mathrm{ml}$ ox-LDL for $24 \mathrm{~h}$; no ox-LDL staining was seen when cells were incubated with $\mathrm{HG}$ without ox-LDL treatment (data not shown); ox-LDL positivity is shown as brown cytoplasmic stain (arrow); original magnification $\times 400$
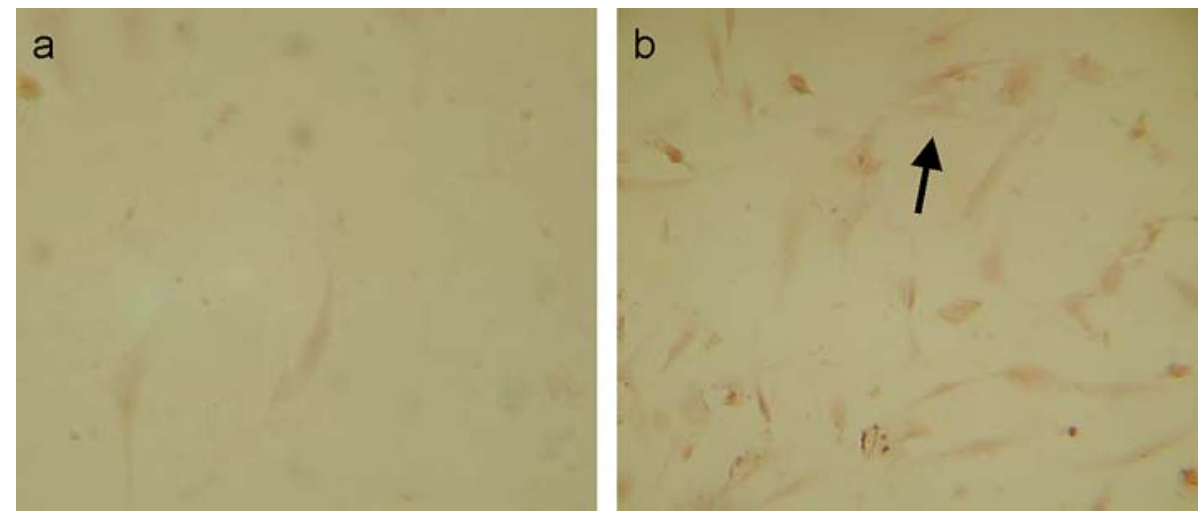

Glucose-induced CD36 up-regulation is associated with increased uptake of ox-LDL and increased oxidative damage We evaluated whether HG-induced up-regulation of CD36 could lead to increased uptake of ox-LDL. Using immunocytochemical analyses, we demonstrated that HG causes significant increase in ox-LDL uptake by ECs as compared with cells cultured in LG (Fig. 3). To characterise the functional significance of increased uptake of ox-LDL, we subjected the cells to real-time RT-PCR analysis with regard to the molecular marker of oxidative stress, HO-1 [27, 28]. Our mRNA data demonstrate that high levels of glucose and ox-LDL increase HO-1 transcript levels (Fig. 4a; $p<0.05$ ). Treatment of cells exposed to $\mathrm{HG}$ with ox-LDL further up-regulated the oxidative stress-response protein, HO-1 (Fig. $4 \mathrm{a} ; p<0.05$ ). Immunocytochemical analysis of oxidative DNA damage also revealed increased 8-OHdG positivity in cells exposed to ox-LDL with $25 \mathrm{mmol} / \mathrm{l}$ glucose but not $5 \mathrm{mmol} / \mathrm{l}$ (data not shown). In parallel to oxidative damage, ox-LDL caused aberrant expression of ET-1, suggesting endothelial dysfunction (Fig. $4 \mathrm{~b} ; p<0.05$ ).

$C D 36$ gene silencing prevents glucose-induced ox- $L D L$ uptake and reduces HO-1 and ET-1 expression To further confirm that HG-induced CD36 up-regulation mediates increased ox-LDL uptake, we assayed for CD36 activity following specific CD36 gene silencing. Sensitive and specific real-time RT-PCR analysis of CD36 mRNA in
Fig. 4 Glucose- and ox-LDLinduced changes in MVECs showing up-regulation of $\mathrm{HO}-1$ (a) and ET-1 (b). HO-1 and ET-1 transcript levels are expressed as the ratio of target to $\beta$-actin (relative to $\mathrm{LG}) ; * p<0.05 \mathrm{HG}$ compared with LG; $n=4$ / treatment a

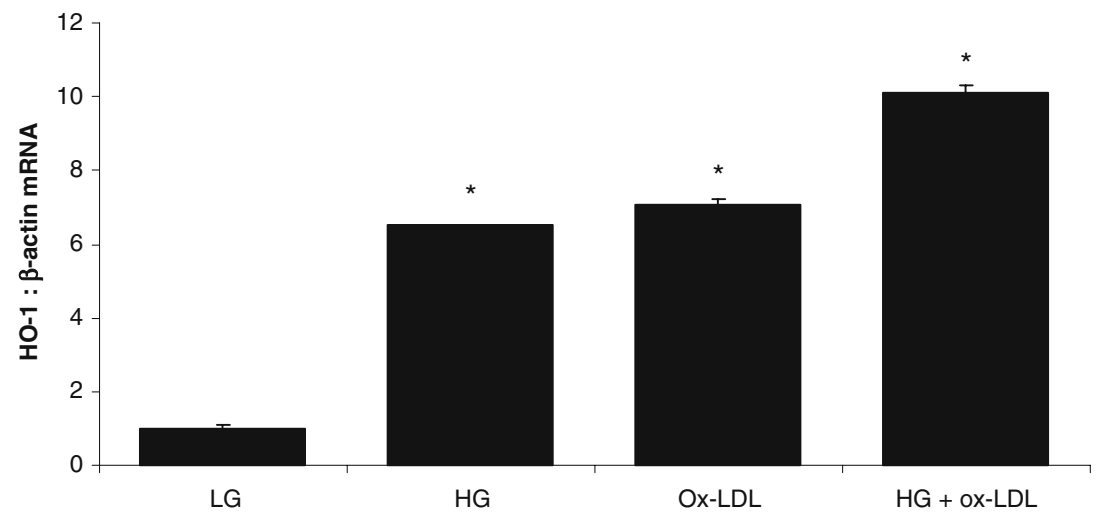

b

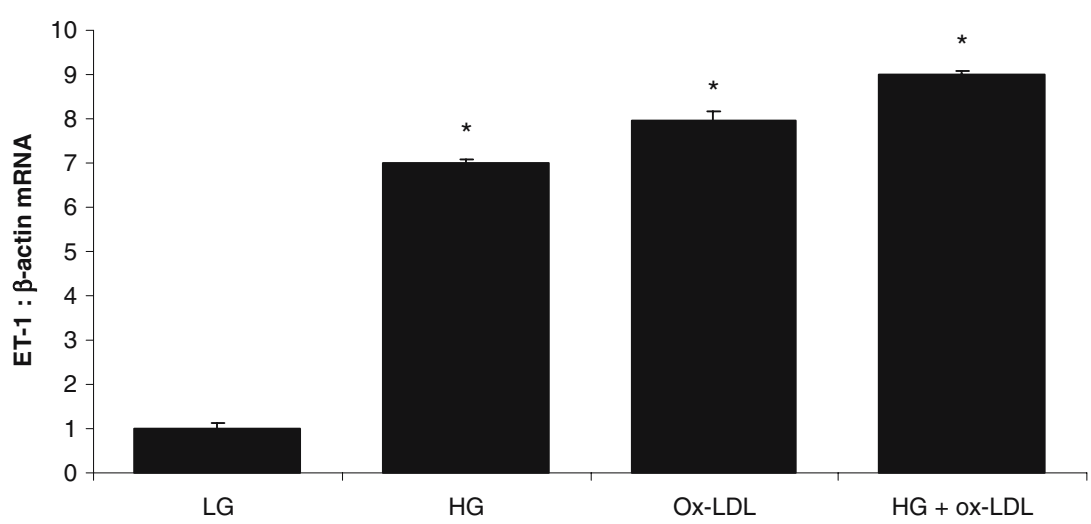


Fig. 5 Effect of CD36 gene silencing on ox-LDL uptake and oxidative stress in MVECs showing a real-time RT-PCR analysis of CD36 mRNA following siRNA transfections, b ox-LDL immunoreactivity in negative control transfected cells, c ox-LDL immunoreactivity in CD36 siRNAtransfected cells and d HO-1 (solid bars) and ET-1 (shaded bars) gene expression changes following CD36 siRNA transfections. CD36 mRNA levels are shown as \% total; ox-LDL immunoreactivity was assayed in cells exposed to $\mathrm{HG}$ and 80 $\mu \mathrm{g} / \mathrm{ml}$; ox-LDL positivity is shown as brown cytoplasmic stain (arrow); original magnification $\times 400$; HO- 1 and ET- 1 mRNA levels are relative to $\mathrm{HG}+$ negative control transfections; ${ }^{*} p<0.05$ compared with negative transfections; $n=4 /$ treatment
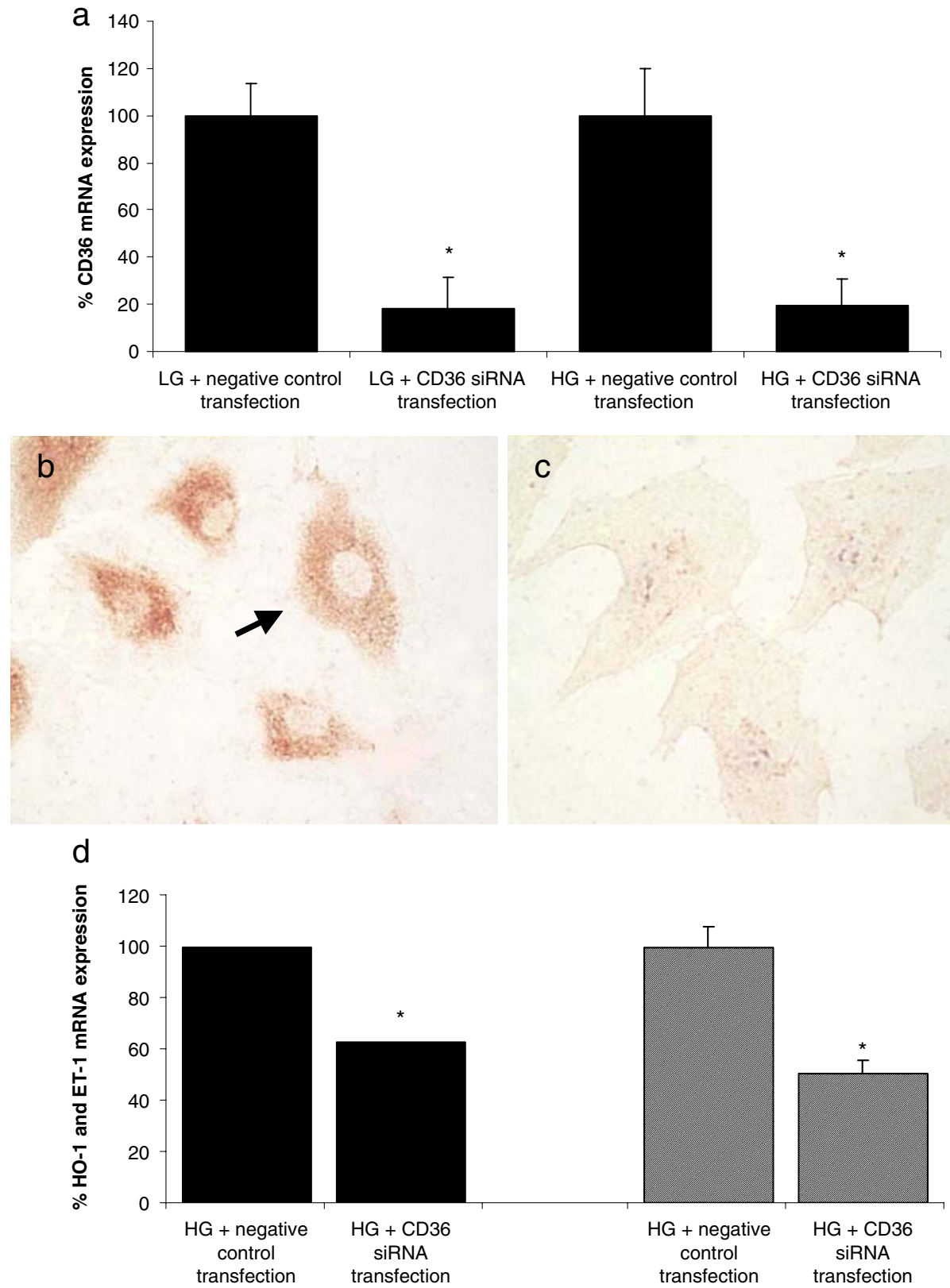

ECs transfected with CD36 siRNA showed greater than $80 \%$ reduction in CD36 transcript levels (Fig. 5a; $p<0.05$ ). CD36 siRNA also prevented HG-induced CD36 upregulation. Following successful transfections, cells were treated with ox-LDL and assayed for uptake by immunohistochemistry and molecular markers of oxidative stress by RT-PCR. Our results show complete normalisation of glucose-induced ox-LDL uptake in CD36 siRNA-transfected cells as compared with negative control transfected cells (Fig. 5b, c). These results indicate that glucose-induced CD36 alteration arbitrates increased ox-LDL uptake in ECs.

We next determined whether CD36 inhibition by siRNA prevents glucose/ox-LDL-induced up-regulation of HO-1 and ET-1. Our data indicate that CD36 siRNA transfection, which reduced CD36 expression by $80 \%$, reduced glucose-induced HO-1 and ET-1 up-regulation (Fig. 5d; $p<0.05)$. CD36 siRNA transfection also reduced HO- 1 and ET-1 mRNA levels in cells exposed to LG (data not shown).

Table 1 Clinical monitoring of animals

\begin{tabular}{lcc}
\hline & Control & Diabetic \\
\hline Blood glucose (mg/dl) & $71.8 \pm 3.6$ & $473.2 \pm 5.9^{*}$ \\
$\mathrm{HbA}_{\mathrm{c}} \mathrm{c}(\%)$ & $4.9 \pm 0.8$ & $17.8 \pm 0.7^{*}$ \\
Body weight $(\mathrm{g})$ & $528.5 \pm 31.4$ & $439.3 \pm 19.8^{*}$ \\
Systolic BP $(\mathrm{mmHg})$ & $107 \pm 6$ & $112 \pm 7$ \\
\hline
\end{tabular}

${ }^{*} p<0.05$ compared with control 
Diabetes induces up-regulation of CD36 and oxidative stress in the heart In order to determine whether diabetes leads to increased oxidative stress via alteration of CD36 receptors, we used a well-established model of chronic diabetes. This STZ-induced diabetic model shows development of myocardial abnormalities similar to humans with diabetes [32]. STZ-induced diabetic animals exhibited hyperglycaemia as compared with non-diabetic controls
(Table 1). Diabetic animals also demonstrated reduced body weight gain and elevated $\mathrm{HbA}_{1} \mathrm{c}$ levels. Systolic BP, recorded by tail plethysmography, did not differ among the animal groups. These parameters are indicative of diabetic dysmetabolism.

Using the real-time RT-PCR assay (Fig. 6a, b), we measured CD36 transcript levels in heart tissues of these diabetic and non-diabetic control animals. Our results in-
Fig. 6 Real-time RT-PCR amplification of rat CD36, showing a PCR amplification curves, b melting curve analysis (MCA) of post-PCR products, and $\mathbf{c}$ quantification of CD36 mRNA in heart tissues. Each trace represents a heart cDNA sample; all PCR reactions were coupled to MCA to determine specificity of amplification; CD36 mRNA is expressed as ratio of target to $\beta$-actin (relative to control); $* p<0.05$ compared with controls; $n=6 /$ group
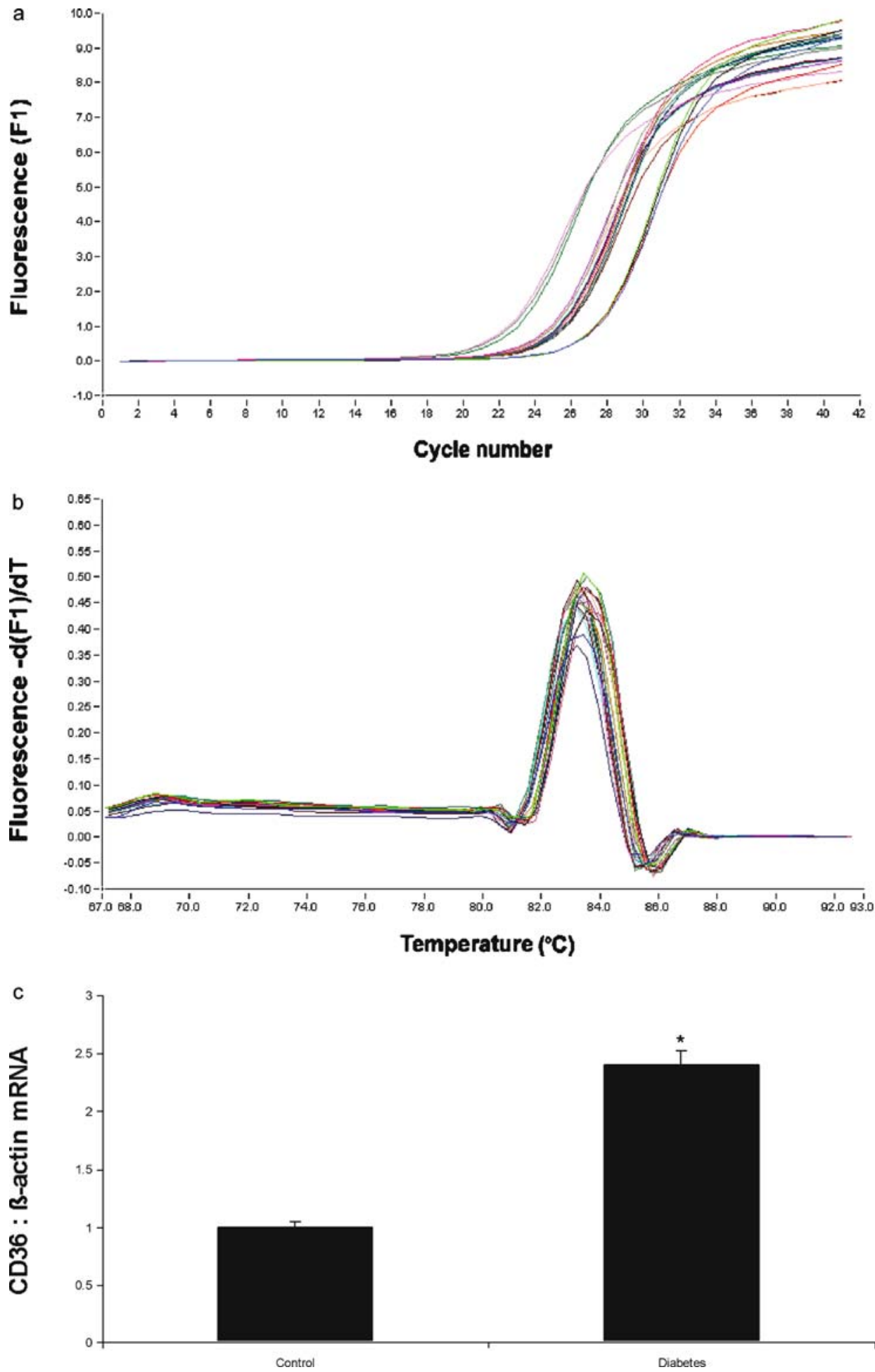
Fig. 7 Oxidative damage in heart tissues of diabetic animals as assessed by $8-\mathrm{OHdG}$ and nitrotyrosine immunoreactivity. a $8-\mathrm{OHdG}$ in control rats, b $8-\mathrm{OHdG}$ in diabetic rats, c nitrotyrosine in control rats, d nitrotyrosine in diabetic rats, and e semi-quantitative analysis of 8-OHdG (solid bars) and nitrotyrosine (shaded bars) immunoreactivity. Positivity is demonstrated as brown nuclear stain for 8-OHdG (arrow) and brown cytoplasmic stain for nitrotyrosine (arrow); original magnification $\times 400$; immunoreactivity was assessed by measuring number of positive cardiomyocytes in ten random fields containing approximately 100 cells; ${ }^{*} p<0.05$ compared with controls
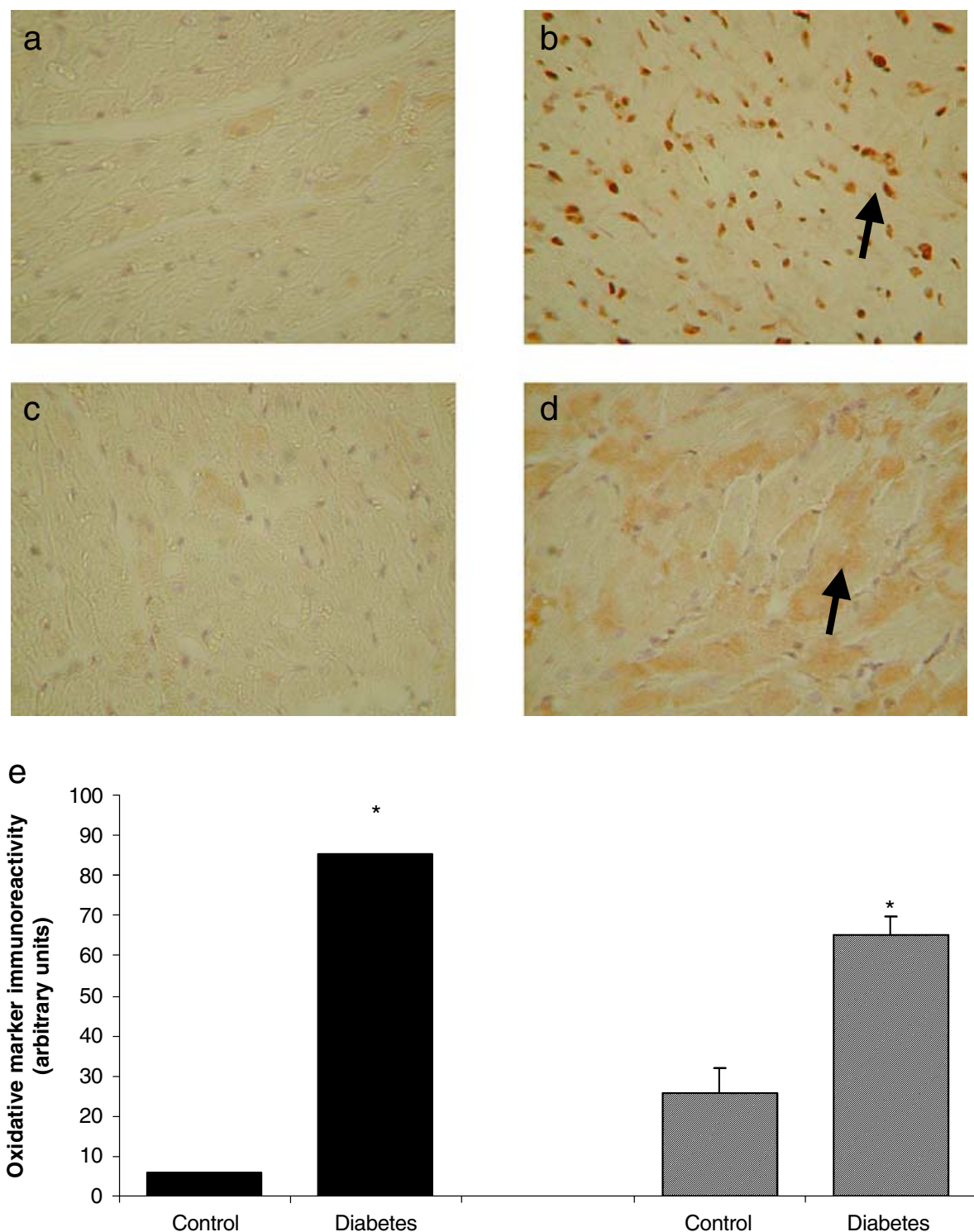

dicate that diabetes leads to up-regulation of CD36 mRNA in the heart (Fig. $6 \mathrm{c} ; p<0.05$ ). These results provide evidence of transcriptional regulation of CD36 in diabetes.

We used 8-OHdG and nitrotyrosine as markers of oxidative damage in the heart tissues. These two markers detect DNA and protein residues modified by oxidation and have been extensively used to provide an index of oxidative damage [30, 33, 34]. Our data confirm previous findings and show increased oxidative DNA and protein damage in the heart tissues of diabetic animals (Fig. 7; $p<0.05)$.

\section{Discussion}

We have shown that cultured MVECs demonstrate a glucose-induced up-regulation of CD36. Such alteration leads to increased uptake of ox-LDL molecules, increased molecular markers of oxidative stress and oxidative DNA damage, and causes vascular endothelial dysfunction, as evident by increased HO-1 and ET-1 expression. To the best of our knowledge, this is the first indication of transcriptional regulation of CD36 by glucose in MVECs. Furthermore, we have demonstrated that diabetes also leads to up-regulation of CD36 at the transcriptional level in the heart. This up-regulation was found to coincide with increased oxidative stress as assessed by oxidative DNA and protein damage in the heart tissues.

Modified proteins such as ox-LDLs and AGEs may interfere with normal functioning of the diabetic vasculature by interacting with specific receptors on vascular cells, including ECs. Glycated LDLs have been shown to be increased in diabetic individuals [15]. Glycation of LDLs also confers increased susceptibility to oxidative modification [12]. It has been demonstrated that glycation of LDL molecules results in impaired uptake by the LDL receptors $[16,17]$. In contrast to native high-affinity receptors, recognition of modified proteins is enhanced by a highcapacity but low-affinity receptor family, the SR family $[16,17]$. Recently, interest in CD36 has been renewed with 
the demonstration that this surface protein is involved in the recognition and internalisation of oxidatively modified proteins $[18,19]$. Our studies further provide evidence that CD36 up-regulation may be involved in increased oxidative stress in the heart and MVECs. The direct role of CD36 in such alteration was characterised following specific CD36 gene silencing. Our data show that inhibition of CD36 production prevents ox-LDL uptake; however, whether similar mechanisms may mediate increased ox-LDL uptake in vivo remain to be determined. It should be noted that CD36-mediated alteration of fatty acid uptake and metabolism may also contribute to the pathogenesis of diabetic cardiomyopathy [35]. Whether such mechanisms also contribute to oxidative stress remains to be determined.

CD36 alteration has previously been shown in vascular preparations and monocytes isolated from diabetic patients [13]. It should be noted that CD36 alteration in monocytes isolated from diabetic patients was only shown to be at the translational level [13]. It has also been shown that monocytes from diabetic patients show a significantly higher level of CD36 expression, which was not changed during further exposure to hyperglycaemic conditions [36]. In this study, we provide the first indication that CD36 is upregulated at the transcriptional level in MVECs exposed to high levels of glucose. Taken together, these findings suggest that in addition to increased uptake of modified protein by monocytes, vascular ECs may suffer oxidative DNA damage via glucose-induced up-regulation of CD36 [37]. We have demonstrated that glucose increases the uptake of ox-LDL in ECs leading to increased oxidative stress and elaboration of vasoactive peptide, ET-1. In parallel to our studies, ox-LDL has been shown to cause oxidative stress in human fibroblasts [37]. In addition, murine models of type 2 diabetes and mice fed a high-fat diet show up-regulation of CD36 expression in cardiac capillary ECs. It should be noted, however, that CD36 expression in these models of type 2 diabetes could be attributed to hyperglycaemia and/or dyslipidaemia. Our studies provide evidence that hyperglycaemia may be the mechanism underlying CD36 alteration in diabetes. In support of such a notion are findings of a recent study which demonstrates increased CD36 mRNA expression in heart tissues of STZ-diabetic animals [35].

In conclusion, CD36 up-regulation may alter properties of vascular ECs. Diabetes-induced up-regulation of CD36 may be involved in increased oxidative stress and diabetic cardiomyopathy. These studies provide insight into the pathogenesis of chronic diabetic complications. Furthermore, these results may provide avenues which could be explored for the development of therapeutic modalities.

Acknowledgements The authors acknowledge grant support from the Canadian Diabetes Association in honour of the late Glenn W. Liebrock, the Canadian Institutes of Health Research, and the Lawson Health Research Institute.

\section{References}

1. Harris MI (1998) Diabetes in America: epidemiology and scope of the problem. Diabetes Care 21:C11-C14

2. International Diabetes Federation (2003) Diabetes atlas, 2nd edn. International Diabetes Federation, Brussels, Belgium

3. Aleksandrovski YA (1998) Molecular mechanisms of diabetic complications. Biochemistry (Mosc) 63:1249-1257

4. Sheetz MJ, King GL (2002) Molecular understanding of hyperglycemia's adverse effects for diabetic complications. JAMA 288:2579-2588

5. Hamby RI, Zoneraich S, Sherman L (1974) Diabetic cardiomyopathy. JAMA 229:1749-1754

6. Rodrigues B, McNeill JH (1992) The diabetic heart: metabolic causes for the development of a cardiomyopathy. Cardiovasc Res 26:913-922

7. Giugliano D, Ceriello A, Paolisso G (1995) Diabetes mellitus, hypertension, and cardiovascular disease: which role for oxidative stress? Metabolism 44:363-368

8. Nishikawa T, Edelstein D, Du XL et al (2000) Normalizing mitochondrial superoxide production blocks three pathways of hyperglycaemic damage. Nature 404:787-790

9. Baynes JW, Thorpe SR (1999) Role of oxidative stress in diabetic complications: a new perspective on an old paradigm. Diabetes 48:1-9

10. Nadler J, Winer L (1996) Free radicals, nitric oxide and diabetic complications. In: LeRoith D, Taylor SI, Olefsky M (eds) Diabetes mellitus. Lippincott-Raven, Philadelphia, PA, pp 840-848

11. Cadenas E (1989) Biochemistry of oxygen toxicity. Annu Rev Biochem 58:79-110

12. Bucala R, Makita Z, Koschinsky T, Cerami A, Vlassara H (1993) Lipid advanced glycosylation: pathway for lipid oxidation in vivo. Proc Natl Acad Sci U S A 90:6434-6438

13. Griffin E, Re A, Hamel N et al (2001) A link between diabetes and atherosclerosis: glucose regulates expression of CD36 at the level of translation. Nat Med 7:840-846

14. Tsuzura S, Ikeda Y, Suehiro T et al (2004) Correlation of plasma oxidized low-density lipoprotein levels to vascular complications and human serum paraoxonase in patients with type 2 diabetes. Metabolism 53:297-302

15. Cohen MP, Lautenslager G, Shea E (1993) Glycated LDL concentrations in non-diabetic and diabetic subjects measured with monoclonal antibodies reactive with glycated apolipoprotein B epitopes. Eur J Clin Chem Clin Biochem 31:707-713

16. Steinbrecher UP, Witztum JL (1984) Glucosylation of lowdensity lipoproteins to an extent comparable to that seen in diabetes slows their catabolism. Diabetes 33:130-134

17. Klein RL, Laimins M, Lopes-Virella MF (1995) Isolation, characterization, and metabolism of the glycated and nonglycated subfractions of low-density lipoproteins isolated from type I diabetic patients and nondiabetic subjects. Diabetes 44:1093-1098

18. Boullier A, Bird DA, Chang MK et al (2001) Scavenger receptors, oxidized LDL, and atherosclerosis. Ann N Y Acad Sci 947:214-222

19. Endemann G, Stanton LW, Madden KS, Bryant CM, White RT, Protter AA (1993) CD36 is a receptor for oxidized low density lipoprotein. J Biol Chem 268:221-236

20. Tandon NN, Lipsky RH, Burgess WH, Jamieson GA (1989) Isolation and characterization of platelet glycoprotein IV (CD36). J Biol Chem 264:7570-7575

21. Abumrad NA, el Maghrabi MR, Amri EZ, Lopez E, Grimaldi PA (1993) Cloning of a rat adipocyte membrane protein implicated in binding or transport of long-chain fatty acids that is induced during preadipocyte differentiation. Homology with human CD36. J Biol Chem 268:17665-17668 
22. Greenwalt DE, Scheck SH, Rhinehart-Jones T (1995) Heart CD36 expression is increased in murine models of diabetes and in mice fed a high fat diet. J Clin Invest 96:1382-1388

23. Miyazaki A, Nakayama H, Horiuchi S (2002) Scavenger receptors that recognize advanced glycation end products. Trends Cardiovasc Med 12:258-262

24. Khan ZA, Cukiernik M, Gonder JR, Chakrabarti S (2004) Oncofetal fibronectin in diabetic retinopathy. Invest Ophthalmol Vis Sci 45:287-295

25. Liu WL, Guo X, Guo ZG (1998) Oxidized low-density lipoproteins induce apoptosis in vascular smooth muscle cells. Zhongguo Yao Li Xue Bao 19:245-247

26. Chow SE, Chu WK, Shih SH, Chen JK (2002) Exposure to oxidized low-density lipoprotein reduces activable Ras protein in vascular endothelial cells. In Vitro Cell Dev Biol Anim $38: 320-325$

27. Kamalvand G, Pinard G, Ali-Khan Z (2003) Heme-oxygenase1 response, a marker of oxidative stress, in a mouse model of AA amyloidosis. Amyloid 10:151-159

28. Tyrrell RM, Basu-Modak S (1994) Transient enhancement of heme oxygenase $1 \mathrm{mRNA}$ accumulation: a marker of oxidative stress to eukaryotic cells. Methods Enzymol 234:224-235

29. Khan ZA, Chakrabarti S (2003) Endothelins in chronic diabetic complications. Can J Physiol Pharm 81:622-634

30. Farhangkhoee H, Khan ZA, Mukherjee S et al (2003) Heme oxygenase in diabetes-induced oxidative stress in the heart. J Mol Cell Cardiol 35:1439-1448
31. Chen S, Khan ZA, Barbin Y, Chakrabarti S (2004) Pro-oxidant role of heme oxygenase in mediating glucose-induced endothelial cell damage. Free Radic Res 38:1301-1310

32. Rodrigues B, McNeill JH (1999) Physiological and pathological consequences of streptozotocin diabetes on the heart. In: McNeill JH (ed) Experimental model of diabetes. CRC Press LLC, Boca Raton, FL, pp 63-80

33. Piconi L, Quagliaro L, Ceriello A (2003) Oxidative stress in diabetes. Clin Chem Lab Med 41:1144-1149

34. Abu-Qare AW, Abou-Donia MB (2001) Combined exposure to sarin and pyridostigmine bromide increased levels of rat urinary 3-nitrotyrosine and 8-hydroxy-2'-deoxyguanosine, biomarkers of oxidative stress. Toxicol Lett 123:51-58

35. Luiken JJ, Arumugam Y, Bell RC et al. (2002) Changes in fatty acid transport and transporters are related to the severity of insulin deficiency. Am J Physiol Endocrinol Metabol 283: E612-E621

36. Sampson MJ, Davies IR, Braschi S, Ivory K, Hughes DA (2003) Increased expression of a scavenger receptor (CD36) in monocytes from subjects with type 2 diabetes. Atherosclerosis 167:129-134

37. Maziere C, Meignotte A, Dantin F, Conte MA, Maziere JC (2000) Oxidized LDL induces an oxidative stress and activates the tumor suppressor p53 in MRC5 human fibroblasts. Biochem Biophys Res Commun 276:718-723 